\title{
Cost effectiveness analysis of larval therapy for leg ulcers
}

\author{
Marta O Soares, research fellow, ${ }^{1}$ Cynthia P Iglesias, senior research fellow, I Martin Bland, professor of \\ health statistics, ${ }^{1}$ Nicky Cullum, professor, deputy head of department, ${ }^{1}$ Jo C Dumville, research fellow, \\ E Andrea Nelson, reader in wound healing and director of research, ${ }^{2}$ David I Torgerson, professor, director \\ York trials unit, ${ }^{1}$ Gill Worthy, trial statistician ${ }^{1}$ on behalf of the VenUS II team
}

\begin{abstract}
${ }^{1}$ Department of Health Sciences, University of York, York Y010 5DD

${ }^{2}$ School of Healthcare, University of Leeds, UK

Correspondence to: $\mathrm{M} O$ Soares ms602@york.ac.uk
\end{abstract}

Cite this as: BMJ 2009;338:b825 doi:10.1136/bmj.b825

\section{ABSTRACT}

Objective To assess the cost effectiveness of larval therapy compared with hydrogel in the management of leg ulcers.

Design Cost effectiveness and cost utility analyses carried out alongside a pragmatic multicentre, randomised, open trial with equal randomisation.

Population Intention to treat population comprising 267 patients with a venous or mixed venous and arterial ulcers with at least $25 \%$ coverage of slough or necrotic tissue. Interventions Patients were randomly allocated to debridement with bagged larvae, loose larvae, or hydrogel.

Main outcome measure The time horizon was 12 months and costs were estimated from the UK National Health Service perspective. Cost effectiveness outcomes are expressed in terms of incremental costs per ulcer-free day (cost effectiveness analysis) and incremental costs per quality adjusted life years (cost utility analysis).

Results The larvae arms were pooled for the main analysis. Treatment with larval therapy cost, on average, $£ 96.70$ (€109.61; \$140.57) more per participant per year (95\% confidence interval $-£ 491.9$ to $£ 685.8$ ) than treatment with hydrogel. Participants treated with larval therapy healed, on average, 2.42 days before those in the hydrogel arm (95\% confidence interval -0.95 to 31 . 91 days) and had a slightly better health related quality of life, as the annual difference in QALYs was 0.011 (95\% confidence interval -0.067 to 0.071 ). However, none of these differences was statistically significant. The incremental cost effectiveness ratio for the base case analysis was estimated at $£ 8826$ per QALY gained and $£ 40$ per ulcer-free day. Considerable uncertainty surrounds the outcome estimates.

Conclusions Debridement of sloughy or necrotic leg ulcers with larval therapy is likely to produce similar health benefits and have similar costs to treatment with hydrogel.

Trial registration Current Controlled Trials

ISRCTN55114812 and National Research Register N0484123692.

\section{INTRODUCTION}

Venous leg ulcers are a highly prevalent and costly chronic condition in the United Kingdom. ${ }^{1}$ In 2004 the Healthcare Commission estimated the annual costs of treatment for leg ulcers at $£ 300 \mathrm{~m}$ (€340m; $\$ 436 \mathrm{~m})$ to $£ 600 \mathrm{~m}$. Nursing time required to manage and treat leg ulcers is the main cost driver in the UK, where patients with leg ulcers often make up a large proportion of community nursing caseloads. ${ }^{2}$ Despite the effectiveness of high compression bandaging for the treatment of venous leg ulcers being well established, ${ }^{3-5}$ not all patients are suitable or willing to wear compression. Furthermore, the healing process is slow and some ulcers never heal - thus the relevance of investigating the potential value of alternative treatments aimed at expediting or enhancing the healing process.

A common belief among healthcare providers is that the presence of necrotic tissue and slough might interfere with wound healing; however, there is no strong evidence to support this theory. ${ }^{6-8}$ In consultation with UK tissue viability nurses we identified that in the UK National Health Service, hydrogel (autolytic technique) can be considered the "standard therapy" for the removal of necrotic tissue. Biological methods for debridement, such as larval therapy, have been proposed as a potentially effective and cost effective method for removing necrotic tissue and are thought to promote healing. Larvae are available in two formulations: bagged and loose. Compared with hydrogel, each application of larvae is expensive ( $£ 58$ for loose larvae, $£ 98.79$ for bagged larvae, $£ 1.55$ for hydrogel) and more nurse consultations are likely to be required for the application and removal of larvae up to debridement. Although larval therapy might clean wounds more quickly than hydrogel it is unclear whether this would result in faster healing and reduced consumption of healthcare resources.

The value for money of larval therapy had been previously investigated only in a partial economic analysis done alongside a randomised controlled trial with 12 patients. Limitations associated with the design of this clinical study as well as the methodology used to explore cost effectiveness compromise the confidence we can have in the previous conclusion that larval therapy economically dominates hydrogel as a treatment for leg ulcers. ${ }^{9}$ Given this uncertainty we carried out an economic evaluation alongside a large multicentre 

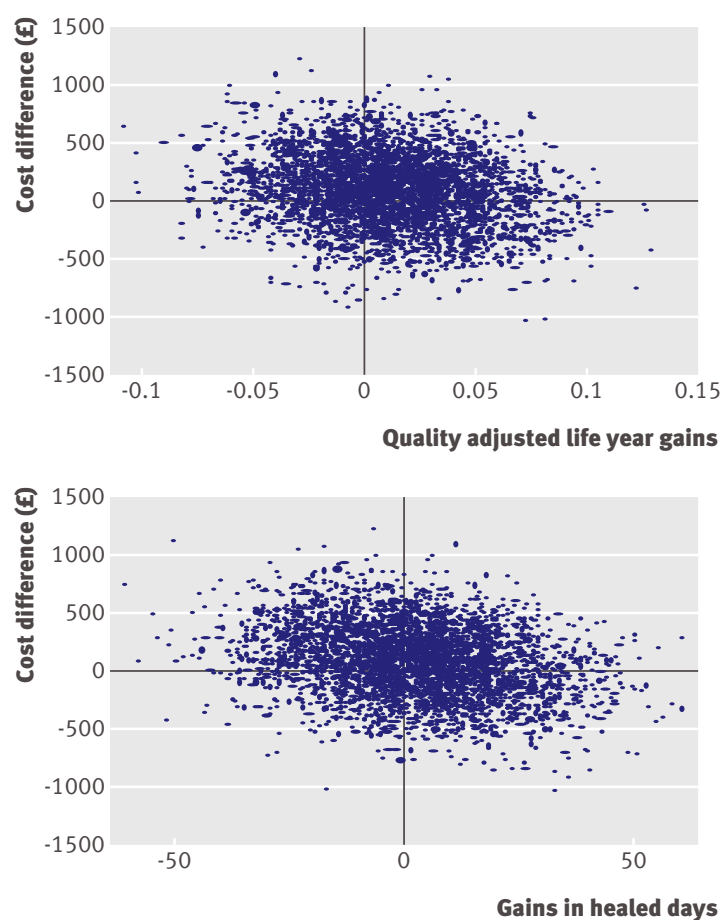

Fig 1| Base case analysis cost effectiveness plane

randomised controlled trial to investigate the cost effectiveness of larval therapy compared with hydrogel in patients with venous or mixed venous and arterial leg ulcers. The clinical results of the trial are published in an accompanying paper. ${ }^{10}$

\section{METHODS}

Full details of the trial are available elsewhere. ${ }^{10}$ Briefly, patients with venous or mixed venous and arterial leg ulcers were eligible for recruitment if one of the ulcers had at least 25\% surface coverage with slough or necrotic tissue. The $25 \%$ cut-off point was adopted on the advice of tissue viability nurses, who indicated that in practice larvae would be considered a suitable treatment only in these circumstances.

\section{Interventions}

Participants were randomised to one of three arms: hydrogel, loose larvae, or bagged larvae. Larvae were left on the ulcer for three or four days, during which time the patients received nursing care for wound assessment and rehydration. On removal of the larvae the treating nurse assessed the amount of slough or necrotic tissue remaining and decided whether a further application was required. We refer to the application of the debriding agents until full debridement or the discontinuation of debridement treatment as the debridement phase. Participants did not receive compression while undergoing larval therapy, as this potentially suffocates the larvae. This trial design therefore relates to the pragmatic question of whether the benefits of larval therapy outweigh the disbenefits of going without compression during larval therapy.

\section{Economic analysis}

We carried out a cost effectiveness and a cost utility analysis using patient level data collected within a randomised controlled trial (the leg ulcer trial, VenUS II). Intention to treat analyses compared incremental costs with incremental ulcer-free days (cost effectiveness analysis) and incremental quality adjusted life years (cost utility analysis). The cost effectiveness analysis was done using time to healing as the outcome measure. Healing was selected as the primary end point since clinicians are motivated to debride wounds because they believe that clean, debrided wounds heal more quickly than non-debrided ones, and yet there is no evidence that this is the case.

VenUS II was funded by the UK NHS and therefore its main objective is to inform decision making in the NHS. In this respect the perspective for the economic evaluations was that of the NHS and Personal Social Services. ${ }^{11}$ The year of pricing was 2006 . The time horizon for the analyses was 12 months after recruitment, and consequently we discounted neither costs nor health benefits. The analyses were done using Stata 10 (StataCorp 2007, TX, USA).

The clinical analysis plan indicated that should evidence of a statistically non-significant difference in debridement time between loose and bagged larvae be found, data from both larval groups would be analysed as a single group - that is, the data from both groups would be pooled. In line with this, the incremental economic analysis considered only two alternative groups: larvae and hydrogel.

\section{Resource use and unit costs}

We collected data on use of resources from nurse completed and participant completed questionnaires. Analysis was carried out using 2006 costs in pounds sterling. For the purposes of costing resource use, the following data were collected:

Debriding agents - Information about the number of pots and units of loose and bagged larvae, respectively, was collected by the treating nurse at each consultation. Similarly, the number of individual hydrogel applications was recorded at each consultation. Unit costs for all debriding agents were obtained from the British National Formulary and larvae suppliers. One pot of loose larvae (LarvE, Zoobiotic, Bridgend, Wales) was costed at $£ 58$ (300 maggots) and a half pot at $£ 35$ (150 maggots, no longer available) plus $£ 16.50$ postage costs in each case. Bagged larvae (Biobag; Biomonde, Barsbüttel, Germany) sachets containing 50, 100,200 , and 300 maggots were costed at, respectively, $£ 59.26$, £67.17, £79.03, and £98.79 plus £20.89 carriage costs for each application. Hydrogel (Purilon Gel; Coloplast, Denmark) was costed at $£ 1.55$ per application. ${ }^{12}$

Healthcare consultations -Data on contacts with nurses and doctors at home, clinics, and hospital were available from both nurse and participant reports. Nurse reported data were systematically collected for only the reference ulcer, whereas participant self reported data were collected for all ulcers and ulcer 
related conditions. In the presence of multiple ulcers participants would continue recording healthcare resource use irrespective of the healing status of the reference ulcer. Participants' data were therefore used in the base case analysis. A distinction was made between the duration of consultations at home and in clinics (table 1). Hospital consultations were costed on an outpatient basis.

Compression therapy-The nature of the compression therapy applied by nurses throughout the trial was recorded at each visit and the costs estimated as the arithmetical average cost for commercially available systems. ${ }^{10}$

Table 1 provides detailed descriptions of the categories of cost and units of measurement. Other dressings and treatments were assumed to be used similarly across treatment arms and are thus not relevant for the incremental analysis. ${ }^{14}$

\section{Health outcomes}

Health benefit was measured in terms of ulcer-free days and quality adjusted life years (QALYs). Time to

\begin{tabular}{|c|c|c|}
\hline Variables & Value & Source \\
\hline \multicolumn{3}{|l|}{ Base case analysis: patient reported data } \\
\hline \multicolumn{3}{|l|}{ Nurse consultations: } \\
\hline Duration of clinic visits (minutes) & 22 & VenUS $I^{5}$ \\
\hline Duration of home visits (minutes) & 40 & VenUS I ${ }^{5}$ \\
\hline \multicolumn{3}{|l|}{ Cost per minute depending on location: } \\
\hline Clinic visits & 0.667 & PSSRU $^{13}$ \\
\hline Home visits & 1.085 & PSSRU $^{13}$ \\
\hline Travelling fixed cost for home visits & 1.3 & PSSRU $^{13}$ \\
\hline \multicolumn{3}{|l|}{ Doctor consultations: } \\
\hline \multicolumn{3}{|l|}{ Cost per doctor visit: } \\
\hline Surgery consultation & 25 & PSSRU $^{13}$ \\
\hline Home visits (including travel time) & 69 & PSSRU $^{13}$ \\
\hline Cost per ulcer related hospital stay (outpatient visit) & 113 & PSSRU $^{13}$ \\
\hline \multicolumn{3}{|l|}{ Sensitivity analysis: nurse reported data* } \\
\hline \multicolumn{3}{|l|}{ Duration of clinic visits, according to task: } \\
\hline Loose larvae application (minutes) & 37 & Survey \\
\hline Bagged larvae application (minutes) & 22.5 & Survey \\
\hline Dressing application (minutes) & 22 & VenUS $I^{5}$ \\
\hline \multicolumn{3}{|l|}{ Duration of home visits, according to task: } \\
\hline Loose larvae application (minutes) & 67.3 & Survey, VenUS I ${ }^{5}$ \\
\hline Bagged larvae application (minutes) & 40.9 & Survey, VenUS ${ }^{5}$ \\
\hline Dressing application (minutes) & 40 & Survey, VenUS ${ }^{5}$ \\
\hline \multicolumn{3}{|l|}{ Cost per minute depending on location: } \\
\hline Clinic visits & 0.667 & PSSRU $^{13}$ \\
\hline Home visits & 1.085 & PSSRU $^{13}$ \\
\hline Travelling fixed cost for home visits & 1.3 & PSSRU $^{13}$ \\
\hline Visits to general practice & 0.467 & PSSRU $^{13}$ \\
\hline Hospital visits & 0.667 & PSSRU $^{13}$ \\
\hline
\end{tabular}

$£ 1.00$ ( $€ 1.13 ; \$ 1.45)$. PSSRU=Personal Social Services Research Unit.

${ }^{\star}$ Costing of nurse consultations for required distinct unit costs owing to differences in data collection

instruments between patient reported and nurse reported data. Doctor and hospital unit costs were considered as detailed for base case analysis. healing of the reference ulcer was recorded by nurses and independently ascertained by blinded investigators using digital photographs. ${ }^{10}$ Health related quality of life data, as the EQ-5D ${ }^{15}$ health state descriptor, were collected at baseline and at three, six, nine, and 12 months. Utility scores were calculated using an independent predefined algorithm obtained by the elicitation of societal preferences for the health dimensions in a random population sample through a time trade-off technique. ${ }^{16}$ Thus time lived in perfect health has a weight of 1 , which decreases as health becomes impaired. Quarterly QALYs were calculated by applying an individual's utility weights to survival time using the area under the curve approach, ${ }^{1718}$ which was defined by linearly interpolating the utility scores measured over time.

\section{Statistical analysis}

Estimates of expected cost and health benefit were reported for larval therapy (pooling data from the loose and bagged larvae groups) and hydrogel. None the less, for completeness we present descriptive measures of costs and health benefits for each of the three trial arms.

We used inverse probability weighting ${ }^{19-21}$ to estimate the mean time to healing, costs, and QALYs, accounting for the censored nature of these data. ${ }^{22}$ The weights were evaluated as the inverse of the Kaplan Meier estimator of censoring probability. For QALYs and cost estimation, we partitioned the study time horizon in homogeneous subintervals (quarterly intervals) through the weighted regression mean cost, and QALYs were estimated within each interval and then summed across intervals to estimate mean total costs and QALYs.

Linear regression was used to adjust the estimates for relevant covariates: type of ulcer, duration of ulcer (logarithmic), ulcer area (logarithmic), and centre (aggregating centres with fewer than 10 participants), in conformity with the clinical analysis. This accounts for possible baseline imbalances and ensures that randomisation stratification variables are appropriately considered in the analysis. We included baseline EQ$5 \mathrm{D}$ scores as a covariate in the estimation of QALYs. ${ }^{17}$

We calculated 95\% confidence intervals for differential costs and effectiveness using non-parametric bootstrap estimates (bias corrected). ${ }^{23}$ For each bootstrap resample we obtained adjusted estimates of expected total costs and effectiveness measures.

A treatment strategy can be considered cost effective only if the decision maker's willingness to pay for an additional unit of health benefit is equal to or greater than the incremental cost effectiveness ratio. The decision maker is responsible for establishing the willingness to pay. Nevertheless, the treatment decision is uncertain because expected costs and effectiveness are estimated under conditions of uncertainty. As decision makers have to decide on the provision of services, we explored this uncertainty using cost effectiveness planes and acceptability curves. ${ }^{24}$ Given the trial data, the acceptability curve for larval therapy 
Table 2 |Characterisation of trial debridement treatment and patient reported resource use*. Values are numbers (percentages) unless stated otherwise

\begin{tabular}{|c|c|c|c|}
\hline Variables & Loose larvae $(n=94)$ & Bagged larvae $(n=86)$ & Hydrogel $(n=87)$ \\
\hline \multicolumn{4}{|c|}{$\begin{array}{l}\text { Time until first treatment } \\
\text { application (days) }\end{array}$} \\
\hline Mean (SD) & $5.09(3.86)$ & $5.61(4.46)$ & $2.49(3.99)$ \\
\hline Missing & $7(7)$ & 7 (8) & $8(9)$ \\
\hline \multicolumn{4}{|c|}{ No of applications of trial treatment: } \\
\hline Mean (SD) & $1.44(1.22)$ & $1.46(1.06)$ & $9.2(27.78)$ \\
\hline Missing & $5(5)$ & $4(5)$ & $5(6)$ \\
\hline \multicolumn{4}{|c|}{ Duration of trial treatment (days): } \\
\hline Mean (SD) & $11.95(9.11)$ & $12.84(11.47)$ & $43.17(51.76)$ \\
\hline Missing & $6(6)$ & $4(5)$ & $9(10)$ \\
\hline \multicolumn{4}{|c|}{$\begin{array}{l}\text { Nurse consultations related to } \\
\text { ulcers: }\end{array}$} \\
\hline Mean (SD) & $37(40)$ & $36(41)$ & $39(45)$ \\
\hline Missing & $6(6)$ & $4(5)$ & $5(6)$ \\
\hline \multicolumn{4}{|c|}{$\begin{array}{l}\text { Doctor consultations related to } \\
\text { ulcers: }\end{array}$} \\
\hline Mean (SD) & $2(4)$ & $3(5)$ & $4(9)$ \\
\hline Missing & $6(6)$ & $4(5)$ & $5(6)$ \\
\hline \multicolumn{4}{|c|}{ Hospital visits related to ulcers: } \\
\hline Mean (SD) & $10(20)$ & $7(15)$ & $5(12)$ \\
\hline Missing & $6(6)$ & $4(5)$ & $5(6)$ \\
\hline \multicolumn{4}{|c|}{ Highest compression level used: } \\
\hline None & $7(7.5)$ & $4(4.7)$ & $1(1.2)$ \\
\hline Low & $18(19.2)$ & $22(25.6)$ & $20(23.0)$ \\
\hline High & $64(68.1)$ & $56(65.1)$ & $61(70.1)$ \\
\hline Missing & $5(5.3)$ & $4(4.7)$ & $5(5.8)$ \\
\hline
\end{tabular}

*Healthcare resource use by treatment arm is further detailed in Dumville et al. ${ }^{10}$

represents the probability of this therapy being cost effective compared with hydrogel for a range of willingness to pay values for an ulcer-free day or QALY. This represents a Bayesian interpretation of uncertainty, although a full Bayesian analysis was not undertaken.

\section{Sensitivity analysis}

Total costs of treating venous leg ulcers are driven by community nursing costs and costs of hospital stay. ${ }^{5} \mathrm{As}$ information on contact with community nurses and hospital visits was also collected by nurses (as well as from participants), we explored the use of this alternative information source in sensitivity analysis. Nurses collected data that allowed their consultations to be costed according to the venue in which these took place: home, clinic, general practice, or hospital. We defined the duration of consultations by the expected duration of treatment application (see table 1). Nurse derived data also distinguished outpatient from inpatient hospital visits; consequently costs of ulcer related hospital stays were based on the duration of hospital stay through a bed day cost. Nevertheless, if a participant was admitted to hospital for a non-ulcer related reason and received ulcer treatment while in hospital, we calculated this ulcer related cost considering only the hospital nurses' time. In the sensitivity analysis, unit costs for doctor consultations and hospital stay were the same as in the base case analysis (table 1).

We also considered an exploratory sensitivity analysis investigating the effect of amputations. As data on amputations were not collected systematically in the clinical trial, the results from this analysis should be interpreted with caution. The full trial report gives a description of the results from this analysis. ${ }^{25}$

\section{RESULTS}

A total of 267 people were recruited into VenUS II: 94 were allocated to loose larvae, 86 to bagged larvae, and 87 to hydrogel. Randomised treatments were adminis tered to $88(94 \%), 82(95 \%)$, and $78(90 \%)$ participants, respectively. Mean follow-up time was 171 days (167 days for the loose larvae group, 170 days for the bagged larvae group, and 175 days for the hydrogel group).

\section{Resource use \\ Debriding agents}

Table 2 describes the number and duration of trial debridement treatments. Participants allocated to larval therapy received their first treatment application about three days later than participants allocated to hydrogel, owing to the need to order the larvae. Participants in either larval therapy arm received on average 1.45 applications before the debridement treatment was discontinued or data were censored compared with on average 9.2 applications in the hydrogel arm.

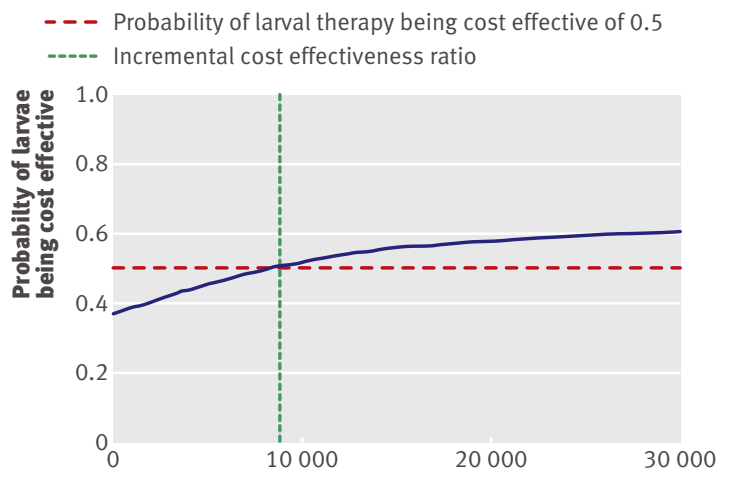

Willingness to pay for one additional quality adjusted life year (f)

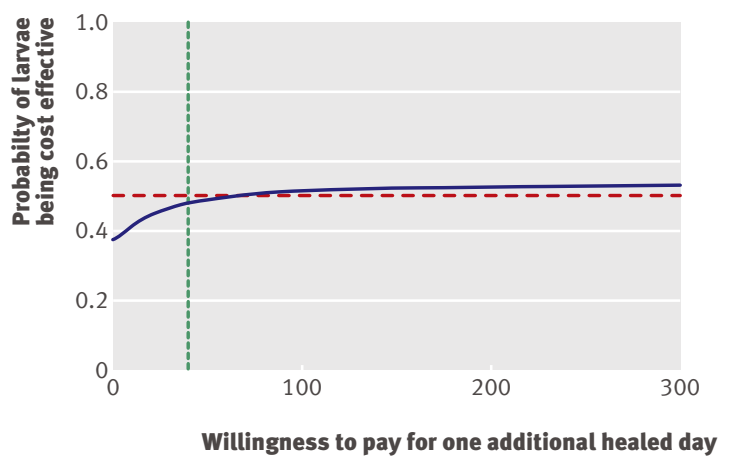

Fig 2 | Base case analysis cost effectiveness acceptability curve 
Table 3 |Adjusted base case analysis*: annual costs ( $£$ ), time to healing, and quality adjusted life years (QALYs)

\begin{tabular}{lccc} 
Variables & \multicolumn{1}{c}{$\begin{array}{c}\text { Mean }(95 \% \mathrm{Cl}) \\
\text { annual costs }\end{array}$} & $\begin{array}{c}\text { Mean }(95 \% \mathrm{Cl}) \text { time to } \\
\text { healing (days) }\end{array}$ & $\begin{array}{c}\text { QALYs } \\
(95 \% \mathrm{Cl})\end{array}$ \\
Hydrogel & $1976.4(1521.4$ to 2500.2$)$ & $206.5(202.7$ to 260.2$)$ & $0.540(0.489$ to 0.589$)$ \\
\hline Larval therapy & $2073.1(1724.4$ to 2433.4$)$ & $204.1(207.9$ to 248.3$)$ & $0.551(0.505$ to 0.591$)$ \\
\hline Difference & $96.7(-491.9$ to 685.8$)$ & $-2.42(-41.0$ to 31.9$)$ & $0.011(-0.067$ to 0.071$)$ \\
\hline
\end{tabular}

$£ 1.00(€ 1.13 ; \$ 1.45)$

*Adjusted for type of ulcer, duration of ulcer (logarithmic), ulcer area (logarithmic), centre (aggregating centres with fewer than 10 elements). Additional adjustment for baseline utility in estimation of QALYs.

tLarval therapy compared with hydrogel.

Nineteen participants never received the allocated treatment: data on nurse visits were missing for 14 participants, whereas five received a treatment other than the trial treatment (one in the loose larvae arm and four in the hydrogel arm). The duration of trial treatment was, on average, 30 days longer in the hydrogel arm than in the larval therapy arms (43 v 12 days).

\section{Healthcare consultations}

Table 2 details the number of patient reported healthcare consultations related to ulcers. The average numbers of total consultations with healthcare professionals in the follow-up period were similar across groups ( 59 for loose larvae, 56 for bagged larvae, and 61 for hydrogel), with most visits being ulcer related $(81 \%$ overall, 47/58). Nurse consultations accounted for $71 \%$ of the total number of healthcare consultations (42/59).

\section{Compression therapy}

The use of high and low compression bandaging (at least once in the trial) was similar across the three arms (table 2): compression was received by $87 \%$ of participants (82/94) in the loose larvae arm, 91\% $(78 / 86)$ in the bagged larvae arm, and 93\% (81/87) in the hydrogel arm.

\section{Total costs}

The average estimated cost of the trial treatment per application was: loose larvae $£ 71.70$ (SD $£ 13.40$; minimum-maximum: £51.50-£132.50), bagged larvae $£ 111.90$ (SD 33.6; £80.10-£218.50), and hydrogel $£ 1.50$ (SD 0).

The mean total unadjusted costs incurred by participants allocated to each of the trial arms was $£ 1833$ (SD $£ 1978$ ) for the loose larvae arm, $£ 1696$ (SD £1948) for the bagged larvae arm, and $£ 1596$ (SD £1861) for the hydrogel arm. The cost of nurse and hospital visits was the major driver of total costs, representing $85 \%$ for loose larvae, $77 \%$ for bagged larvae, and $82 \%$ of the total unadjusted costs of patients allocated to hydrogel. Half of the mean incurred costs observed during the trial (available case analysis) were incurred during the first three months of follow-up in all treatment arms. The analysis adjusted for censoring, baseline imbalances, and stratification variables shows that treatment with larvae costs, on average, $£ 96.70$ more per participant per year (95\% confidence interval $-£ 491.90$ to $£ 685.80$ ) than treatment with hydrogel (table 3). This difference was not statistically significant.

\section{Health outcomes}

On average and after adjustment for baseline imbalances and stratification variables, participants treated with larval therapy healed 2.42 days before those in the hydrogel arm. However, this difference was not

Table 4 | Unadjusted utility weights (EQ-5D) by arm and time. Values are numbers (percentages) unless stated otherwise

\begin{tabular}{|c|c|c|c|}
\hline Follow-up & Loose larvae $(n=94)$ & Bagged larvae $(n=86)$ & Hydrogel $(n=87)$ \\
\hline \multicolumn{4}{|l|}{ Baseline: } \\
\hline Mean (SD) & $0.534(0.301)$ & $0.434(0.342)$ & $0.539(0.313)$ \\
\hline Median & $0.648(-0.239$ to 1$)$ & $0.587(-0.349$ to 1$)$ & $0.62(-0.239$ to 1$)$ \\
\hline Missing & $8(9)$ & $5(6)$ & $6(7)$ \\
\hline \multicolumn{4}{|l|}{3 months: } \\
\hline Mean (SD) & $0.551(0.343)$ & $0.562(0.33)$ & $0.559(0.317)$ \\
\hline Median & $0.620(-0.594$ to 1$)$ & $0.620(-0.349$ to 1$)$ & $0.620(-0.181$ to 1$)$ \\
\hline Missing & $23(24)$ & $18(21)$ & $16(18)$ \\
\hline \multicolumn{4}{|l|}{6 months: } \\
\hline Mean (SD) & $0.596(0.334)$ & $0.588(0.339)$ & $0.566(0.301)$ \\
\hline Median & $0.691(-0.594$ to 1$)$ & $0.587(-0.349$ to 1$)$ & $0.689(-0.181$ to 1$)$ \\
\hline Missing & $33(35)$ & $25(29)$ & $26(30)$ \\
\hline \multicolumn{4}{|l|}{9 months: } \\
\hline Mean (SD) & $0.608(0.345)$ & $0.561(0.381)$ & $0.628(0.315)$ \\
\hline Median & $0.691(-0.594$ to 1$)$ & $0.620(-0.239$ to 1$)$ & $0.691(-0.349$ to 1$)$ \\
\hline Missing & $41(44)$ & $33(38)$ & $38(44)$ \\
\hline \multicolumn{4}{|l|}{12 months: } \\
\hline Mean (SD) & $0.630(0.329)$ & $0.565(0.382)$ & $0.615(0.322)$ \\
\hline Median & $0.691(-0.594$ to 1$)$ & $0.620(-0.181$ to 1$)$ & $0.674(-0.239$ to 1$)$ \\
\hline Missing & $47(50)$ & $41(48)$ & $43(49)$ \\
\hline
\end{tabular}


Table 5|Adjusted sensitivity analysis*

\begin{tabular}{|c|c|}
\hline Arm & Scenario: annual costs $(£)(95 \% \mathrm{Cl})$ \\
\hline Hydrogel & 2369.9 (1773.6 to 3004.7$)$ \\
\hline Larval therapy & 2338.7 (1964.5 to 2719.1) \\
\hline Difference $†$ & $-31.3(-726.0$ to 707.9$)$ \\
\hline \multicolumn{2}{|c|}{$\begin{array}{l}\text { *Adjusted for type of ulcer, duration of ulcer (logarithmic), ulcer area } \\
\text { (logarithmic), centre (aggregating centres with fewer than } 10 \text { elements). } \\
\text { †Larval therapy compared with hydrogel. }\end{array}$} \\
\hline
\end{tabular}

statistically significant $(95 \%$ confidence interval -40.95 days to 31.91 days; see table 3 ).

Table 4 presents the quarterly utility scores per participant by trial arm. The adjusted results show that patients in the larval therapy arms had, on average, a slightly better quality of life than those in the hydrogel arm (annual difference in QALYs 0.011, 95\% confidence interval -0.067 to 0.071 ; see table 3 ).

\section{Cost effectiveness and associated uncertainty}

The incremental cost effectiveness ratio associated with use of larval therapy was estimated at $£ 8826$ per QALY gained and $£ 40$ per ulcer-free day. The point estimates of cost and effect differences were small relative to their standard error, indicating considerable uncertainty associated with the decision to adopt larval therapy (see table 3).

Despite the point estimate for the incremental costutility ratio (£8826) being below the $£ 30000$ per QALY that is generally accepted as being a "threshold" of cost effectiveness by the National Institute for Health and Clinical Excellence, considerable uncertainty surrounds this estimate. This uncertainty can be displayed in two ways. In the cost effectiveness plane (fig 1), 4000 bootstrapped incremental costs and utilities are scattered to represent the joint uncertainty distribution. A similar density is seen across all quadrants of the plane, meaning that the probability of larvae being either cost saving and more effective than hydrogel or more costly and less effective are similar. The cost effectiveness acceptability curve (fig 2), plotted for a range of willingness to pay thresholds (cost per QALY), suggests that in the base case analysis the probability of larvae being cost effective in relation to hydrogel never exceeds $63 \%$.

\section{Sensitivity analysis}

A sensitivity analysis was done to assess the impact on the cost effectiveness estimates of using nurse reported data on consultations rather than patient reported data, as in the base case analysis. The number of consultations and hospital stays (nurse reported) was slightly lower than that reported by patients, but similar across the three arms (38 nurse visits per patient in each arm and a total number of seven ulcer related hospital stays in patients allocated to loose larvae, four allocated to bagged larvae, and two allocated to hydrogel). This may be because nurses did not continue to collect data on resource use after the reference ulcer had healed. The use of nurse reported data allowed us to distinguish between hospital consultations and inpatient stays and to cost them appropriately.

After adjustment for baseline imbalances and stratification variables, cost estimates from the sensitivity analysis indicated lower costs were incurred by larvae users. On average participants in the larvae group cost $£ 31.30$ less than those in the hydrogel group; however, this difference was not statistically significant (table 5). As only costs are subjected to sensitivity analysis, the cost effectiveness or utility was based on the health benefits estimates of the base case analysis. The decision to adopt larval therapy was still associated with considerable uncertainty (fig 3).

\section{DISCUSSION}

Our base case analysis indicates that, compared with hydrogel, larval therapy confers a small health benefit for people with leg ulcers, as measured by QALYs and time to healing, at a minor additional cost to the UK NHS. The incremental cost effectiveness ratio for the base case analysis was estimated at $£ 8826$ per QALY gained and $£ 40$ per ulcer-free day. Yet our non-parametric confidence intervals indicated a high level of uncertainty associated with the differential cost, effectiveness, and cost effectiveness of larval therapy compared with hydrogel. The spread of points on the cost effectiveness plane was almost uniform over the four quadrants, suggesting that the nature of the uncertainty associated with our results is such that larval therapy is likely to be as costly and as effective as hydrogel.

- - - Probability of larval therapy being cost effective of 0.5

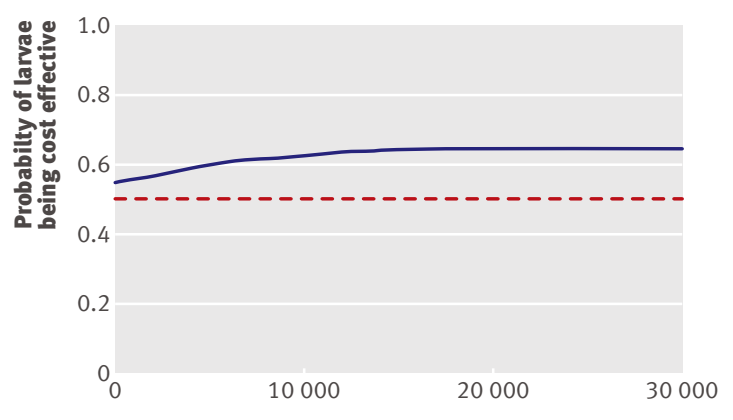

Willingness to pay for one additional quality adjusted life year (f)

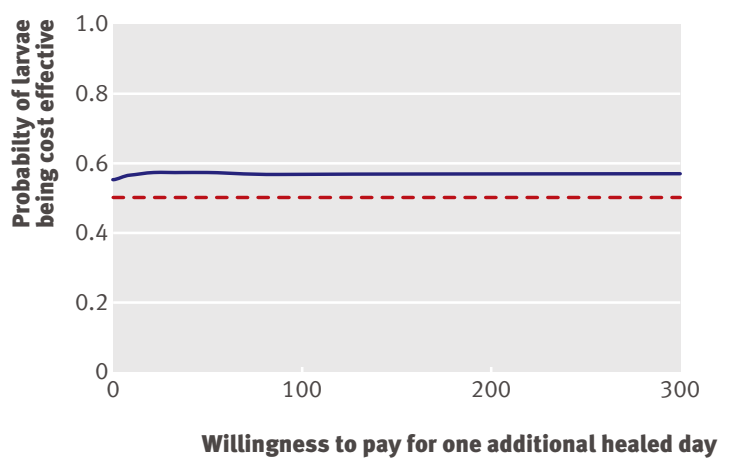

Fig 3 | Sensitivity analysis cost effectiveness acceptability curve 


\section{Exploration of uncertainty}

Sensitivity analysis showed that the uncertainty could not be resolved by using alternative data to estimate the number of contacts with healthcare providers. While in this analysis the point estimate for cost effectiveness suggested "dominance" of larval therapy — that is, larval therapy being better and cheaper than hydrogelthe uncertainty associated with this result was consistent with the base case analysis suggesting no difference between larval therapy and hydrogel in terms of health benefits or costs.

\section{Debridement and health related quality of life}

The impact of larval therapy on health related quality of life (as measured through the EQ-5D and SF-12 $2^{10}$ ) is unclear. As a consequence of the high levels of morbidity in patients with leg ulcers, it could be argued that generic health related quality of life instruments might not capture the benefits of ulcer treatments. Previous work, however, showed that both the SF-12 and EQ-5D are sensitive to, and thus able to measure, changes in healing status of patients with venous leg ulcer. ${ }^{26}$ That said, the influence of a clean ulcer on health related quality of life has never been studied. Although debridement was more rapid with larval therapy, measurement of its effect on health related quality of life might have been hindered by some factors. Health related quality of life data were collected at quarterly intervals whereas the median time for debridement in the larvae groups was between 14 and 28 days $^{10}$; this time gap might have interfered with the instrument's ability to capture any small changes in health related quality of life. Interestingly, in a qualitative study carried out as part of our randomised controlled trial (VenUS II), a relatively quick reappearance of necrotic tissue was reported by previous users of larval therapy, suggesting that any positive effects in health related quality of life associated with larval therapy may be short lived. In addition, larval therapy was associated with increased levels of pain, ${ }^{10}$ and therefore if the time between debridement and reappearance of slough is short then any potential benefits on health related quality of life associated with larval therapy may be cancelled out.

\section{Data characteristics}

An important characteristic of our dataset was the high proportion of censored data. Heavy censoring is not unusual in studies focusing on patients with high morbidity and frequent transfers between hospital and community healthcare settings. Baseline health related quality of life scores indicated a higher than average level of morbidity among the population under evaluation, which may account for more than $40 \%$ of participants failing to complete the health related quality of life questionnaires at the final follow-up. Inverse probability weighting methods were used to account for this important feature of our data in the estimation of mean health benefits and costs.

In this cost effectiveness analysis, healing was the event of interest; individuals lost to follow-up were censored as were those who died. As healing cannot occur after death, censoring in such cases may conflict with the assumption common to most methods of survival analysis - that is, that censoring is independent of the outcome (non-informative censoring). The impact of this approach to censoring could be explored using multistate models. ${ }^{27}$

\section{Strengths and weaknesses of the economic analysis}

This is the first full economic evaluation alongside a randomised controlled trial evaluating the value for money of a single phase of larval therapy (loose or bagged) compared with hydrogel for the debridement and healing of venous and mixed aetiology leg ulcers over a period of 12 months. The only existing analysis is a partial cost consequences analysis with a one month time horizon and an intermediate outcomedebridement — as a measure of health benefit. ${ }^{9}$

Leg ulcers are a recurrent condition, and consequently it could be argued that to ascertain fully the expected value for money of debriding agents, an economic analysis based on a comprehensive decision analytic model should be done. Given the limited knowledge about the role and value of debridement in the healing process and the relatively rare prevalence of large amounts of necrotic tissue in leg ulcers (indicated by the results of the eligibility screening for this trial) we restricted the time horizon of this analysis to the duration of the trial.

While our findings have strong external validity for the UK NHS, the applicability of these results to other settings worldwide may require further consideration. Variations on the use of debriding agents may have an impact on the cost effectiveness of these treatments.

\section{Recommendations for future research}

In practice several other methods are available for debriding wounds, and thus when making choices about type of debridement treatment decision makers are faced with a more complex decision than that represented by this trial. To make an informed decision from this wide selection of treatment options to ensure that the most cost effective treatments are used, data from this randomised controlled trial and other studies should be incorporated into decision analytic models.

The potential relevance of important adverse events such as amputations may warrant further analysis. ${ }^{25}$ While there is no evidence of a differential rate of amputations between these debridement treatments, given the substantial clinical and economic implications of this event future studies on leg ulcer should systematically collect data on amputations to be included in economic analyses.

\section{Conclusions}

In conclusion, one phase of larval therapy used until initial debridement of leg ulcers is likely to produce a similar level of health benefit at a similar cost to hydrogel. It could be argued that healthcare decision makers should be indifferent when recommending these two 


\section{WHAT IS ALREADY KNOWN ON THIS TOPIC}

Larval therapy, a traditional approach to wound management, is widely used on leg ulcers

Only one randomised trial with 12 participants has been carried out previously and did not measure ulcer healing or do a full cost effectiveness analysis

\section{WHAT THIS STUDY ADDS}

Larval therapy for the debridement of sloughy or necrotic leg ulcers is likely to have similar cost effectiveness to hydrogel

Healthcare decision makers should generally be indifferent when recommending these two treatments

The choice of treatment may then be driven by patients' wishes and experiences of pain with larvae

therapies for the treatment of sloughy or necrotic leg ulcers or that the decision should be driven by the goal of treatment (for example, if debriding an ulcer rapidly is important when a skin graft is planned). The choice of treatment may then be driven by patients' wishes and experiences of pain with larvae.

We thank the participants for taking part in the trial; the research nurses, tissue viability teams, district nurses, and hospital outpatient staff for recruiting participants and completing the trial documentation; the principal investigators at each site for coordinating recruitment of the participants; members of the trial steering committee (Su Mason (chair) Mike Campbell, and Francine Cheater); and members of the data monitoring and ethics committee (Keith Abrams (chair), Michelle Briggs, and Alun Davies) for overseeing the study.

The VenUS II collaborators (current and past) are: Una Adderley, Jacqui Ashton, Gill Bennett, JMB, Anne Marie Brown, Sue Collins, Ben Cross, NC, Val Douglas, CD, JCD, Andrea Ellis, Caroline Graham, Christine Hodgson, Gemma Hancock, Shervanthi Homer-Vanniasinkam, Cl, June Jones, Nicky Kimpton, Dorothy McCaughan, Elizabeth McGinnis, Jeremy Miles, JLM, Veronica Morton, EAN, Sue O'Meara, Angie Oswald, Emily Petherick, Ann Potter, Pauline Raynor, Linda Russell, Jane Stevens, MS, Nikki Stubbs, DJT, Kath Vowden, Peter Vowden, Michael Walker, Shernaz Walton, Val Wadsworth, Margaret Wallace Judith Watson, Anne Witherow, and GW. Contributors: MOS carried out the economic analysis, contributed to both clinical and economic analysis plans, and is guarantor. CPI contributed to the trial design, oversaw the conduct of the economic analysis, and contributed to the design of both economic and clinical analyses. JCD was trial manager between 2005 and 2008 and also contributed to the economic analysis. GW and JMB designed and carried out the clinical analysis. NC was the chief investigator, led the design of the trial, chaired the Trial Management Group, and edited and approved the final draft of the report. EAN and DJT contributed to the design and coordination of the study.

Funding: This project was funded by the UK National Institute for Health Research Health Technology Assessment Programme (project No 01/41/ 04). The views and opinions expressed therein are those of the authors and do not necessarily reflect those of the Department of Health. Zoobiotic supplied and distributed the loose larvae at no cost and Biomonde supplied the bagged larvae at no cost. These manufacturers had no role in the design of the trial or in the collection, analysis, and interpretation of the data.

Competing interests: None declared.

Ethical approval: This study was approved by the West Midlands multicentre research ethics committee and local ethics committees.

1 Nelzen O, Bergqvist D, Lindhagen A. Venous and non-venous leg ulcers: clinical history and appearance in a population study. $\mathrm{Br}$ Surg 1994;81:182-7.
2 Callam MJ, Ruckley CV, Harper DR, Dale JJ. Chronic ulceration of the leg: extent of the problem and provision of care. $B M J$ 1985;290:1855-6.

3 Cullum N, Nelson EA, Flemming K, Sheldon T. Systematic reviews of wound care management: (5) beds; (6) compression; (7) laser therapy, therapeutic ultrasound, electrotherapy and electromagnetic therapy. Health Technol Assess 2001;5:1-221.

4 Cullum N, Nelson EA, Fletcher AW, Sheldon TA. Compression for venous leg ulcers. [update of Cochrane Database Syst Rev 2000;(3): CD000265]. Cochrane Database Syst Rev 2001(2):CD000265.

5 Iglesias C, Nelson EA, Cullum NA, Torgerson DJ, Ven UST. VenUS I: a randomised controlled trial of two types of bandage for treating venous leg ulcers. Health Technol Assess 2004;8(29):iii.

6 Bradley M, Cullum N, Sheldon T. The debridement of chronic wounds: a systematic review. Health Technol Assess 3(17 Pt 1):iii-iv.

7 Royal College of Nursing. The nursing management of venous leg ulcers. London: Royal College of Nursing, 2006.

8 Scottish Intercollegiate Guidelines Network. The care of patients with chronic leg ulcers. Edinburgh: SIGN Secretariat, 1998.

9 Wayman J, Nirojogi V, Walker A, Sowinski A, Walker MA. The cost effectiveness of larval therapy in venous ulcers. J Tissue Viability 2000;10:91-4.

10 Dumville J, Worthy G, Soares M, Bland JM, Cullum N, Dowson C, et al. Larval therapy for leg ulcers (VenUS II): randomised controlled trial, BMJ 2009;338:b773.

11 National Institute for Health and Clinical Excellence. NICE technical guidance for manufacturers and sponsors on making a submission to a technology appraisal. London: NICE, 2001.

12 British Medical Association, Royal Pharmaceutical Society of Great Britain. British national formulary. London: BMA, RPS 2006:825-38. (No 52.)

13 Curtis L Netten A. Unit costs of health and social care. Canterbury, University of Kent: Personal Social Services Research Unit, 2006.

14 Drummond M, Sculpher M, Torrance G, O’Brien B, Stoddart G. Methods for the economic evaluation of health care programmes. $3 \mathrm{rd}$ edn. Oxford: Oxford University Press, 2005.

15 Kind PT. The EuroQol instrument: an index of health-related quality of life. In: Spilker B, ed. Quality of life and pharmacoeconomics in clinical trials. Philadelphia: Lippincott-Raven, 1996.

16 Dolan P, Gudex C, Kind P,Williams A. A social tariff for EuroQol: results from a UK general population survey. Discussion paper series (No 138). York: Centre for Health Economics, 1995.

17 Manca A, Hawkins N, Sculpher MJ. Estimating mean QALYs in trialbased cost-effectiveness analysis: the importance of controlling for baseline utility. Health Econ 2005;14:487-96.

18 Matthews JN, Altman DG, Campbell MJ, Royston P. Analysis of serial measurements in medical research. BMJ 1990;300:230-5

19 Zhao H, Tsiatis AA. A consistent estimator for the distribution of quality adjusted survival time. Biometrika 1997;84:339-48.

20 Lin DY. Linear regression analysis of censored medical costs. Biostatistics 2000;1:35-47.

21 Willan AR, Lin DY, Manca A. Regression methods for costeffectiveness analysis with censored data. Stat Med 2005;24:131-45.

22 Willan AR, Lin DY, Cook RJ, Chen EB. Using inverse-weighting in costeffectiveness analysis with censored data. Stat Methods Med Res 2002;11:539-51.

23 Efron B, Tibshirani R, J. An introduction to the bootstrap. London: Chapman and Hall, 1993.

24 Fenwick E, O’Brien BJ, Briggs A. Cost-effectiveness acceptability curves-facts, fallacies and frequently asked questions. Health Econ 2004;13:405-15.

25 Dumville J, Worthy G, Soares M, Bland JM, Cullum N, Dowson C, et al. VenUS II: A randomised controlled trial of larval therapy in the management of leg ulcers. Health Technol Assess (in press).

26 Iglesias CP, Birks Y, Nelson EA, Scanlon E, Cullum NA. Quality of life of people with venous leg ulcers: a comparison of the discriminative and responsive characteristics of two generic and a disease specific instruments. Qual Life Res 2005;14:1705-18

27 Gardiner IC, Luo Z, Bradley CI, Sirbu CM, Given CW. A dynamic model for estimating changes in health status and costs. Stat Med 2006;25:3648-67.

Accepted: 28 January 2009 